\title{
Le collège doctoral franco-allemand « Construire les différences - Unterschiede denken »
}

\section{Hartmut Kaelble et Iris Schröder}

Traducteur : Jean-Louis Georget

URL : http://journals.openedition.org/ifha/420

DOI : 10.4000/ifha.420

ISSN : 2198-8943

Éditeur

IFRA - Institut franco-allemand (sciences historiques et sociales)

Édition imprimée

Date de publication : 30 septembre 2012

Pagination : 116-123

ISSN : 2190-0078

Référence électronique

Hartmut Kaelble et Iris Schröder, « Le collège doctoral franco-allemand « Construire les différences Unterschiede denken » », Revue de l'IFHA [En ligne], 4 | 2012, mis en ligne le 14 février 2013, consulté le 20 avril 2019. URL : http://journals.openedition.org/ifha/420 ; DOI : 10.4000/ifha.420

Ce document a été généré automatiquement le 20 avril 2019.

(C)IFHA 


\title{
Le collège doctoral franco-allemand «Construire les différences - Unterschiede denken »
}

\author{
Hartmut Kaelble et Iris Schröder \\ Traduction : Jean-Louis Georget
}

Le collège doctoral franco-allemand "Construire les différences - Unterschiede denken ", financé par l'Université franco-allemande et mis en place en 2006, achèvera la deuxième phase de son existence en 2012. De 2006 à 2009, le thème central en a été « La dynamique des représentations dans la formation de la modernité européenne - Die Dynamik der Repräsentationen in der Formierung der europäischen Moderne» - et de 2009 à 2012 «Construire les différences - Unterschiede denken ». Le collège a pour ambition, à travers les thématiques évoquées, d'explorer d'une part le vaste champ de l'histoire des différences et des ressemblances franco-allemandes, des imbrications et des transferts, des transgressions de frontières disciplinaires et des passerelles, des autoreprésentations et des appréhensions de l'altérité, mais également dans le même temps de débattre lors des rencontres entre chercheurs français et allemands des analogies et divergences touchant les méthodes et la terminologie scientifique, l'utilisation des théories et des sources. La thématique avait donc un double objectif: il s'agissait d'une structure commune pour les projets de thèse de doctorat et d'une plate-forme appropriée pour un travail commun à l'intérieur du collège.

Le collège doctoral est porté à la fois par l'École des hautes études en sciences sociales à Paris et l'université Humboldt à Berlin. Participent également au collège des directeurs de recherche du CNRS, des enseignants-chercheurs des universités Paris-I, Paris-IV et ParisXIII, des chercheurs du Centre Marc Bloch à Berlin, de l'université technique de Berlin, de l'université libre de Berlin et du Wissenschaftszentrum Berlin für Sozialforschung (WZB). Les enseignants-chercheurs et les doctorants du collège viennent d'horizons disciplinaires divers : histoire médiévale, moderne et contemporaine, histoire de l'art et ethnologie. Le collège comprend 25 doctorants et 14 enseignants-chercheurs. La demande d'inscription de la part de doctorants a été et reste encore élevée. Le collège doctoral a été coordonné 
et dirigé dans sa première phase du côté parisien par Pierre Monnet (qui a quitté l'équipe de coordination après sa nomination comme président de l'Université franco-allemande), Michael Werner et Jean-Claude Schmitt, puis dans sa seconde phase par Falk Bretschneider et Christophe Duhamelle, du côté berlinois dans ses deux phases par Iris Schröder et Hartmut Kaelble. Parmi les enseignants-chercheurs du collège doctoral on comptait entre autres Horst Bredekamp, Étienne François, Jean-Louis Georget, Dieter Gosewinkel, Johannes Helmrath, Wolfgang Kaschuba, Anne Kwaschik, Gérard Laudin, Rebekka von Malinckrodt, Gabriele Metzler, Alexander Nützenadel, Bénédicte Savoy, Laure Schnapper, Daniel Schönpflug, Maria Stavrinaki, Claudia Ulbrich, Patrice Veit et Bénédicte Zimmermann. Il est tout à fait significatif que, dans ce contexte, les lignes de partage nationales soient brouillées. En effet, dans le groupe parisien des enseignantschercheurs et doctorants, on comptait plusieurs Allemands tandis que dans le groupe berlinois semblable, on dénombrait des chercheurs français. Les deux groupes ne se différenciaient plus par la possession de passeports différents, mais par le lieu de travail effectif. Le but du collège doctoral est d'arriver à créer un échange scientifique entre les doctorants et les encadrants parisiens et berlinois issus des trois disciplines du collège, et, en outre, à faire financer les voyages dans les archives et les séjours de recherche des doctorants parisiens et berlinois dans la ville partenaire. Le collège doctoral ne possède pas de moyens propres pour octroyer des bourses doctorales complètes, mais propose aux doctorants des compléments matériels pour les déplacements nécessaires dans l'autre capitale (jusqu'à 18 mois de mobilité par doctorant à raison de 600 euros par mois de séjour dans le pays partenaire). Du côté français, le collège a pu bénéficier de l'accès aux allocations doctorales fléchées à l'international par le ministère de l'Enseignement supérieur et de la Recherche dans le cadre de son accord avec l'Université francoallemande. Pendant les première et deuxième phases du collège, des allocations doctorales de ce type ont pu être accordées à des doctorants de l'École des hautes études en sciences sociales. Par ailleurs, pendant la première phase de 2006 à 2009, ce sont environ une trentaine de bourses de mobilité doctorale de durée variable qui ont été accordées.

Pourquoi a-t-il été utile pour les enseignants-chercheurs et les doctorants d'investir beaucoup de temps et d'énergie dans ce collège doctoral franco-allemand pendant les six années passées? Il va de soi que le collège doctoral s'est développé progressivement durant la période évoquée à travers l'échange d'expériences mutuelles entre les enseignants et les étudiants, mais aussi de manière concomitante entre les deux pays. La structure possède aujourd'hui un profil très différent de celui des collèges gradués traditionnels, comme par exemple ceux de la Deutsche Forschungsgemeinschaft ou des fondations allemandes, chargés de promouvoir une formation doctorale traditionnelle dans un domaine particulier de la recherche ou voués à soutenir des programmes doctoraux internationaux.

À la différence de tous ces programmes doctoraux, le collège doctoral «Construire les différences - Unterschiede denken » a été doté de manière beaucoup plus faible au plan financier et ne correspond pas à un programme fondamental de recherche qui s'établit entre les doctorants et les enseignants-chercheurs, mais apporte un atout complémentaire : celui d'un axe thématique central qui fédère des projets de doctorat bilatéraux. Les particularités et les mérites du collège doctoral se sont dévoilés peu à peu au fil des rencontres. L'Université franco-allemande s'est adaptée de manière extrêmement flexible et pertinente à cette évolution progressive. 
L'événement central du collège doctoral a consisté dans l'atelier annuel de deux jours, alternativement à Paris et à Berlin. Tant les doctorants que les enseignants-chercheurs devaient s'impliquer obligatoirement dans la préparation de cet atelier annuel pour rencontrer le succès escompté. Deux formats d'ateliers ont été développés, dont le second a surtout été utilisé dans la seconde phase du projet pour atteindre des objectifs précis. La prise de connaissance des projets de thèse et leur commentaire par des doctorants plus avancés et des enseignants-chercheurs ont été l'objet central du colloque de la première année, puisque la plupart des doctorants en étaient encore au début de la rédaction de leur thèse. La discussion sur le contenu personnel de ce qu'ils souhaitaient produire revêtait encore dans ce contexte beaucoup de signification pour eux. Dans le cours plus avancé de la seconde phase du collège, un autre format de colloque, plus approprié aux nouveaux enjeux de travail, a été développé. Au centre des préoccupations ainsi circonscrites, trois thèmes choisis d'un commun accord par les enseignants-chercheurs et les doctorants émergèrent : en 2011 émergèrent «Le statut de l'image en histoire et en histoire de l'art », «La question de la césure » et " Classer, rassembler, distinguer »; pour 2012 "Histoire globale », «Histoire et politique » et «Langue et traduction ». Pour aborder ces thèmes, des textes français et allemands fondamentaux ont été choisis comme autant d'invitations à la réflexion. Chaque doctorant devait se décider pour l'un des sujets proposés, expliquer le sens de son sujet de thèse dans un court résumé de quelques pages au regard du thème choisi et prendre position par rapport aux textes de référence.

À partir du choix des thèmes opéré par les doctorants, des groupes de travail ont été formés, puis ont débattu pendant une demi-journée pour rédiger une synthèse collective, qui était aussi une prise de position par rapport aux documents distribués. Outre le commentaire d'un des enseignants-chercheurs, cette discussion constituait le préambule de la discussion thématique en réunion plénière. Grâce à la préparation intensive de tous les acteurs, les débats ont toujours été de bonne facture et de haut niveau. De plus, les ateliers annuels ont toujours commencé par une manifestation à laquelle les anciens étudiants du collège sont toujours traditionnellement conviés. En 2010, s'est tenus au centre Marc Bloch à Berlin une discussion avec le journaliste et historien Michael Jeismann sur l'importance du doctorat pour la carrière ultérieure, en 2011 à l'Institut historique allemand de Paris un débat sur les avantages et les inconvénients d'un doctorat franco-allemand sous la forme d'une cotutelle, en 2012 une visite guidée du Musée de l'histoire allemande à Berlin, en compagnie d'experts du musée qui avaient pour leitmotiv de leur intervention les trois thèmes du colloque. L'après-midi s'est achevé par un débat public sur le projet qu'était la Maison de l'histoire de France, aujourd'hui abandonné.

Les rencontres annuelles ont été très bénéfiques pour tous les participants. Au final, le collège doctoral a formé un lieu de rencontre important pour les différentes matières représentées, qui, sinon, ne bénéficient que rarement de pouvoir participer à un échange scientifique interdisciplinaire. C'est ainsi que se sont tenues dans le cadre du collège des discussions intenses entre les historiens de l'art et les historiens, des discussions sur la représentation iconographique et le texte ainsi que sur les méthodes d'interprétation et de compréhension différentes de l'histoire de l'art et de l'histoire, tout cela sur l'arrièreplan divergent des évolutions disciplinaires en France et en Allemagne. En outre, un échange soutenu, trop rare entre les experts de différentes périodes historiques, a pu se concrétiser, surtout entre les médiévistes et les modernistes, ce qui a représenté pour 
tous les participants un apport essentiel. Les colloques n'ont pas été seulement destinés aux doctorants, mais également aux enseignants-chercheurs et furent pour eux une expérience exceptionnellement riche, car les différences franco-allemandes en termes de langue scientifique et de méthodes ont été discutées avec une vivacité et une profondeur que l'on trouve rarement ailleurs. L'une des particularités décisives de cette rencontre annuelle, très appréciée de tous, a résidé, au fil du temps, dans une implication accrue des doctorants dans son organisation. Seuls le format et les axes thématiques centraux ont été choisis d'un commun accord avec les enseignants-chercheurs, le reste de l'organisation dans son ensemble, y compris la répartition des doctorants dans les différents axes thématiques, étant pris en charge par les doctorants eux-mêmes. Les comités d'organisation de trois à cinq doctorants issus des deux pays ont investi chaque année beaucoup de temps et d'énergie pour le bon fonctionnement du collège, mais ont acquis par là-même des expériences importantes, assurément utiles ensuite dans leur vie professionnelle.

À côté des rencontres annuelles, qui ont surtout apporté leur substantifique moelle à l'échange intellectuel et scientifique, un autre but important du collège a été d'encourager les jeunes chercheurs à la mobilité dans les archives et aux séjours de recherche. Le collège doctoral s'est donné pour but de faire des séjours des doctorants dans l'autre pays plus qu'un banal voyage pour aller consulter des archives. En effet, le collège doctoral ne devait pas simplement servir à financer des séjours classiques et informels dans les archives. C'est pour cette raison que chaque séjour a été l'occasion de fournir un aperçu du paysage de la recherche de la ville du pays partenaire. Dans cet état d'esprit, les coordinateurs du collège et un doctorant ont revêtu la fonction de tuteurs pour les étudiants venus de l'autre capitale et qui étaient leurs hôtes. Ils leur ont permis de rencontrer les experts de leur sujet de thèse, d'aller à des colloques et des manifestations et d'entrer également en contact avec leurs condisciples sur place. Pour prendre l'exemple de Berlin, nombre de rencontres ont été organisées par les doctorants eux-mêmes, toujours au début d'une nouvelle phase du collège doctoral et afin de se familiariser avec quelques-unes des institutions scientifiques les plus emblématiques de la capitale allemande. Outre une visite au centre Marc Bloch et au centre de recherches françaises, ils furent accueillis dans les locaux de la revue H-Soz-Kult. À partir de ces rencontres formelles, d'autres plus informelles se sont organisées à l'initiative des doctorants eux-mêmes, sans qu'elles aient besoin de faire l'objet d'un quelconque enregistrement.

Pour finir, le collège doctoral franco-allemand s'efforce toujours de procurer aux projets de thèse l'écho scientifique public dont ils ont absolument besoin. Cela passait surtout par la page Web du collège doctoral, qui s'est améliorée au cours du temps. Ce sont là que les projets de thèse ont été présentés, mais également les séminaires et les membres du collège doctoral, afin de les faire connaître parmi les spécialistes de leur domaine [http:// www.differences-differenzen.eu/(11.6. 2012)]. En outre, le site sert aux doctorants pour leurs échanges propres et est consulté également par les anciens étudiants du collège qui souhaitent rester au courant de son actualité.

Le collège doctoral franco-allemand est également un enfant de notre temps. Il profite de trois tendances à l'œuvre dans les relations scientifiques franco-allemandes: le parallélisme croissant des champs de recherche historiques et de la disparition de l'époque où il existait de grandes disparités thématiques dans la discipline, puisqu'en France, on s'attachait avant tout à l'époque de la première modernité, tandis qu'en 
Allemagne on privilégiait le XXe siècle. Le collège profite aussi d'un paradoxe singulier : le recul de l'apprentissage de la langue allemande et française dans le pays voisin, mais concomitamment une amélioration qualitative remarquable des connaissances de la langue de l'autre parmi les doctorants. Le collège doctoral bénéficie directement de la responsabilité commune et durable que la France et l'Allemagne entretiennent envers la construction européenne et à laquelle les relations franco-allemandes bilatérales telles qu'elles existent confèrent une signification remarquable.

Mais un tel collège doctoral franco-allemand est également soumis à l'air du temps. L'intérêt pour les différences franco-allemandes s'amenuise pour plusieurs raisons: l'émergence de nouvelles puissances comme la Chine, l'Inde, le Brésil, la Russie et l'Afrique du Sud fait apparaître des différences avec ces pays bien plus conséquentes que celles qui existent entre la France et l'Allemagne. Avec l'accroissement considérable des compétences de l'Union européenne, l'intérêt pour la politique européenne s'aiguise, mais celui pour ses différents membres s'amenuise en conséquence. De plus, dans le monde anglo-saxon, les relations franco-allemandes ont traditionnellement la réputation d'être une réminiscence de la vieille Europe. Face à la mondialisation, les différences franco-allemandes perdent de leur aura, dans la mesure où la France et l'Allemagne sont touchées de la même façon et apparaissent comme des acteurs globaux dont les profils se ressemblent. Avec l'élargissement de l'Union européenne, l'opinion publique française s'intéresse plutôt à l'espace méditerranéen, l'opinion publique allemande regarde plutôt vers la partie orientale de l'Europe : de fait, l'une et l'autre perdent de leur enthousiasme pour les différences franco-allemandes. De plus, avec l'actuelle crise de la dette, les différences entre l'Europe septentrionale et méridionale refont leur apparition. Dans ce cadre, les différences franco-allemandes ont aussi tendance à être reléguées à l'arrièreplan. Rétrospectivement, le collège doctoral a été constitué en 2006 dans ce mélange de défis et de freins différents de ceux d'aujourd'hui, mais encore plus dans un contexte politique qui n'a rien à voir avec celui qui prévalait dans les années 1980, lorsque le comité d'historiens franco-allemands et les rencontres des spécialistes franco-allemands d'histoire sociale furent fondés comme autant de nouvelles plates-formes du dialogue scientifique entre les historiens français et allemands.

Le collège doctoral franco-allemand entre aujourd'hui dans une troisième phase qui ira de 2013 à 2015. Pour cette période, la thématique générale est conservée : les nouveaux accents sont portés sur la structure sociale, la normativité et la communication. Elle mise sur les interactions et les transferts, et la comparaison est maintenue, mais les relations globales de la France et de l'Allemagne seront mieux prises en compte. Sur le plan disciplinaire, le collège doctoral sera étendu au droit et aux sciences sociales. L'organisation du collège sera légèrement modifiée. Outre les rencontres annuelles, des ateliers en petits groupes réuniront quelques enseignants-chercheurs, chaque doctorant étant finalement associé à un tuteur. De la sorte, les relations entre enseignantschercheurs et doctorants en sortiront intensifiées. En outre, la coopération avec les institutions extra-universitaires sera renforcée et les débats de l'atelier annuel seront plus souvent publiés qu'actuellement. La coordination du collège échoira à d'autres personnes : du côté parisien, elle ira à Rainer Maria Kiesow et Falk Bretschneider, du côté berlinois à Gabriele Metzler et Peter Burschel.

Ce collège doctoral franco-allemand "Construire les différences - Unterschiede denken " doit à l'Université franco-allemande à la fois son financement généreux et sa flexibilité. Le succès du collège s'appuie pour une grande partie sur une coopération étroite avec 
d'autres institutions scientifiques, avant tout le CIERA, le CRIA, l'Institut historique allemand de Paris, le Centre Marc Bloch à Berlin, l'université Humboldt de Berlin et son département spécial de recherche 640 «Représentation des ordres sociaux en transformation ", avec l'Institut français d'histoire en Allemagne, mais surtout avec les personnalités qui portent ces institutions. Pour finir, le succès du collège repose avant tout sur les doctorants. Grâce à leur travail et leur engagement, ils ont fait du collège ce qu'il est aujourd'hui. Beaucoup d'entre eux apprécient le temps qu'ils y ont passé, même après l'avoir quitté. De la sorte, ce sera un devoir et une tâche supplémentaires des trois années à venir que de bien intégrer les doctorants qui vont y travailler, mais également ses anciens bénéficiaires pour continuer de manière fructueuse les activités du collège.

\section{AUTEURS}

\section{HARTMUT KAELBLE}

Harmut Kaelble est professeur émérite de l'université Humboldt

\section{IRIS SCHRÖDER}

Iris Schröder maîtresse de conférences à l'université de Braunschweig pour l'année universitaire 2012-13. Elle a été responsable de l'École doctorale franco-allemande entre l'université Humboldt et l'EHESS du côté allemand dans sa première phase. 